\title{
Very deep spectroscopy of NGC 7009
}

\author{
Xuan Fang ${ }^{1}$, Xiaowei Liu ${ }^{1,2}$ and Peter J. Storey ${ }^{3}$ \\ ${ }^{1}$ Department of Astronomy, School of Physics, Peking University, Beijing 100871, P. R. China \\ email: fangx@pku.edu.cn \\ ${ }^{2}$ Kavli Institute for Astronomy and Astrophysics, Peking University, \\ Beijing 100871, P. R. China \\ ${ }^{3}$ Department of Physics and Astronomy, University College London, \\ Gower Street, London WC1E 6BT, UK
}

\begin{abstract}
We report new calculations of the effective recombination coefficients for the nebular $\mathrm{N}$ II and $\mathrm{O}$ II lines and very deep spectroscopy of the bright planetary nebula (PN) NGC 7009.
\end{abstract}

Keywords. atomic data, line: formation, ISM: abundances, planetary nebulae: individual

New ab initio calculations of the effective recombination coefficients for the $\mathrm{N}$ II and O II recombination spectra were carried out in intermediate coupling by Fang, Storey \& Liu (2011) and P. J. Storey (unpublished), respectively. Both calculations have taken into account the density dependence of effective recombination coefficients arising from the distribution of population among the ground fine-structure levels of the recombining ions. Also new is including the effects of dielectronic recombination via high- $n$ resonances lying between the ground-term thresholds of the recombining ions. The two calculations are valid down to unprecedentedly low temperatures (e.g. $\sim 100 \mathrm{~K}$ for $\mathrm{N} \mathrm{II}$ ), and allow plasma diagnostics based on the N II and O II optical recombination lines (ORLs).

Very deep spectroscopy of planetary nebula NGC 7009 (Fang \& Liu 2011; Fang \& Liu 2012 , in preparation) yields very rich ORLs of heavy element ions. Accurate fluxes of the ORLs of $\mathrm{C}$ II, N II, O II and Ne II were obtained using multi-Gaussian profile fits. The electron temperatures derived from the $\mathrm{N}$ II and $\mathrm{O}$ II ORLs, using the new effective recombination coefficients, were close to $1000 \mathrm{~K}$, which is lower than those derived from the collisionally excited lines (CELs) by nearly one order of magnitude, indicating that the heavy element ORLs originate from very cold region. Various plasma diagnostics yield a temperature sequence, $T_{\mathrm{e}}(\mathrm{CELs}) \gtrsim T_{\mathrm{e}}(\mathrm{H}$ I BJ $) \gtrsim T_{\mathrm{e}}(\mathrm{He}$ I $) \gtrsim T_{\mathrm{e}}(\mathrm{N}$ II \& O II ORLs $)$, which is consistent with the predictions from the bi-abundance nebular model postulated by Liu et al. (2000). The $\mathrm{C}^{2+} / \mathrm{H}^{+}, \mathrm{N}^{2+} / \mathrm{H}^{+}, \mathrm{O}^{2+} / \mathrm{H}^{+}$and $\mathrm{Ne}^{2+} / \mathrm{H}^{+}$ionic abundance ratios derived from ORLs (at $T_{\mathrm{e}}=1000 \mathrm{~K}$ ) are consistently higher, by about a factor of 5 , than the corresponding values derived from CELs, an abundance discrepancy first found for this object by Liu et al. (1995) from deep spectroscopy. For N II, as well as for O II, the ionic abundances derived from different fine-structure components of a multiplet, or from the transitions of different multiplets, agree with each other, indicating that the current theoretical treatment of the recombination spectrum of $\mathrm{N}$ II and $\mathrm{O}$ II is reliable.

\section{References}

Fang, X. \& Liu, X.-W. 2011, MNRAS 415, 181

Fang, X., Storey, P. J., \& Liu, X.-W. 2011, A $3 A$ 530, A18

Liu, X.-W., Storey, P. J., Barlow, M. J., \& Clegg, R. E. S. 1995, MNRAS 272, 369

Liu, X.-W., Storey, P. J., Barlow, M. J., Danziger, I. J., Cohen, M., \& Bryce, M. 2000, MNRAS 312,585 